\title{
Diseño de un instrumento para la cauterización del cervix con dispositivo intrauterino in situ.
}

Dr. SAULO MUÑOZ DELGADO*

La cervicitis crónica con todas sus complicaciones sigue constituyendo una de las causas más frecuentes de consulta tanto en la práctica privada, como en el medio hospitalario. Significa un problema de grandes implicaciones para el futuro reproductivo de las pacientes que desean planificar mediante dispositivo intrauterino.

En estos casos se presenta una situación bastante difícil, pues en el intervalo de tiempo que transcurre para el tratamiento del cervix puede presentarse el embarazo que se pretendía evitar y en el caso de tratar el cervix se pueden quemar los hilos del dispositivo. Además la aplicación del dispositivo en presencia de cervicitis puede originar enfermedad inflamatoria pélvica que posteriormente lleva a discusiones sobre morbilidad atribuible al método. Por tales razones se hacía necesario diseñar y elaborar un instrumento que permitiera tratar adecuadamente el cuello en los casos donde fue necesario aplicar el dispositivo en presencia de cervicitis.

En los casos de usuarias de dispositivo intra-uterino con cervicitis la cauterización del cervix no es posible por el temor de

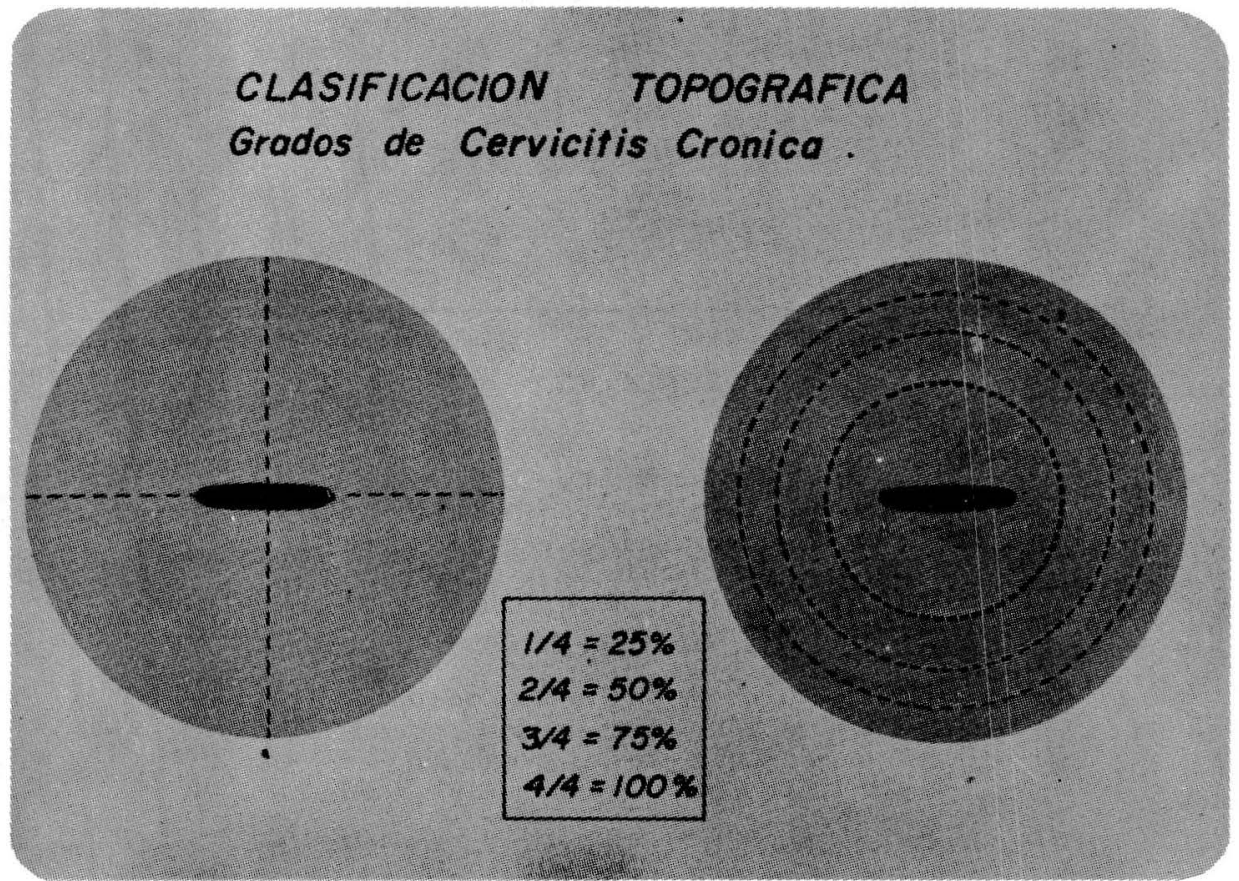

* PROFESOR

AREA MATERNO INFANTIL

FACULT AD DE MEDICINA

UNIVERSIDAD LIBRE

* Director

PROFAMILIA CALI quemar los hilos de Nylon lo que haría necesario practicar dilatación y extracción de este en un medio quirúrgico y bajo anestesia. Con el instrumento diseñado se trató a las pacientes sin tener que retirar el dispositivo. 


\section{MATERIAL Y METODOS:}

Se tomaron pacientes de consultorio privado (S.M.D.) en el transcurso de un (1) año, Junio 1976 a Junio 1977, a quienes se les aplicó dispositivo intrauterino, $\bullet \sin$ tratamiento previo de la cervicitis. Luego se citaron y con el instrumental diseñado (SAMUDEL) se les practicó las cauterizaciones necesarias.

Se fijaron criterios del grado de la cervicitis de acuerdo al de extensión de la lesión dividiéndola en cuartos, ya sea en forma concéntrica o en cuadrantes.

Antes y después de la inserción del dispositivo intra-uterino, se practicó examen pélvico y se clasificó el proceso inflamatorio en grados teniendo en cuenta el dolor como signo de intensidad del proceso, además del compromiso del anexo.

$\begin{array}{ll}+ & \begin{array}{l}\text { Dolor del exámen pélvi- } \\ \text { co }\end{array} \\ ++ & \begin{array}{l}\text { Dolor al examen bima- } \\ \text { nual }\end{array} \\ +++ & \begin{array}{l}\text { Dolor espontáneo } \\ \text { Dolor tipo cólico }\end{array}\end{array}$

\section{RESULTADOS:}

\begin{tabular}{|lc|}
\hline $\begin{array}{l}\text { Cervicitis } \\
\text { Crónica }\end{array}$ & $800=70 \%$ \\
\hline TOTAL & 1.200 \\
\hline
\end{tabular}

No. 1 Pacientes con cervicitis en una muestra continua de consulta privada.

\begin{tabular}{|cccc|}
\hline+ & ++ & ++ & ++++ \\
\hline 25 & 90 & 75 & 25 \\
\hline
\end{tabular}

No. 3 Pacientes con dolor y tratamiento de cervicitis y dispositivo intra-uterino

\begin{tabular}{|cccc|}
\hline+ & ++ & +++ & +++ \\
\hline 30 & 25 & 12 & 3 \\
\hline
\end{tabular}

No. 4.Pacientes con proceso inflamatorio pélvico después de tratamiento de cervicitis.

\section{CONCLUSIONES:}

El instrumento diseñado (SAMUDEL) permite el tratamiento completo de la cervicitis crónica en pacientes en los cuales tengan que aplicarle el dispositivo intrauterino,

En todos los casos fue posible preservar la integridad de los hilos del dispositivo intra-uterino.

A pesar de los cuidados para el tratamiento de la cervicitis en nuestra serie se registraron $29.4 \%$ procesos inflamatorios pélvicos dentro de un seguimiento de 45 a 60 días, recibieron tratamiento adecuado y no presentaron complicación adicional.

\begin{tabular}{|c|c|c|c|c|c|}
\hline & 0 & $I=25$ & $\mathrm{II}=50$ & $\mathrm{III}=75$ & $\mathrm{IV}=100$ \\
\hline $\begin{array}{l}\text { Cervicitis } \\
\text { Crónica y } \\
\text { DIU }\end{array}$ & 30 & 30 & 45 & 95 & 70 \\
\hline
\end{tabular}

TOTAL

270

No. 2 Pacientes a quienes se aplicó dispositivo intrauterino en presencia de cervicitis.

(VI.76 a VI.77) 


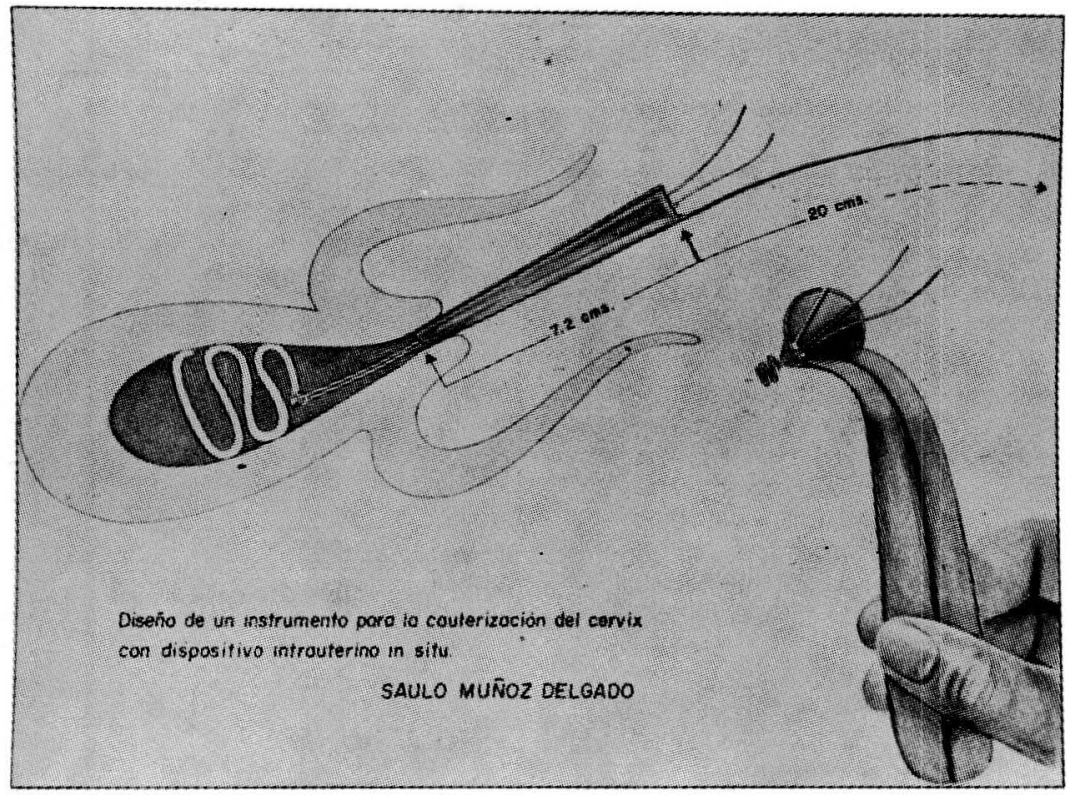

\section{RESUMEN}

El autor presenta el problema de aplicación de DIU en pacientes con cervicitis crónica no tratada y sus complicaciones médicas y procedimentales para la práctica de electrocoagula ción o cauterización. Para obviar el problema, propone un nuevo instrumento que protege del cauterio los hilos de control del dispositivo.

\section{SUMMARY}

The author poses the problem of pla- cing the IUD in pacients with untreated cronic cervisitis and its medical and procedural complication for electrocoagulation or cauterization prac tise. In order to obviate the problem he proposes $a_{r}$ new instrument which protect the devise control wires from cauterization.

\section{BIB LIO G R A F IA}

FLUHMANN C.F; THE CERVIX UTERI-And Its Diseases

W.B. Saunders Company - Philadelphia 1961 London. 